\title{
Development of National Information System (NIS) for sustainable management of biodiversity
}

\author{
P. Shanmughavel*, P. Kumar \\ Department of Bioinformatics, Bharathiar University, Coimbatore, India \\ *e-mail: shanmughavel@buc.edu.in
}

Key words: National Information System (NIS), document and conserve biodiversity, sustainable management of biological resources

Motivation and Aim: The need to document and conserve biodiversity has become a necessity in the wake of increased threats from deforestation, alteration in land use, soil degradation, pollution and climatic change. Current electronic inventories provide limited information on biodiversity, its environmental status, bioclimatic, evolutionary history and management units of conservation [1,2]. This paper explains a system that provides species-specific and site-specific information with improved genetic data semantics to help sustainable management of biological resources. The functions and features of NBGIS are also given.

Methods: NIS can be developed by the authors provides detailed information on bio resources and their environment. The approach employed is to map independently the environmental attributes of a biological species and its spatial distribution over space and time and then integrate them with community data [1,2]. The major tasks proposed in the global initiative for biodiversity conservation is to document floral, faunal and microbial diversity with detailed accounts of species of all regions and developing an integrated interactive information system.

Results: The developed NIS found to be useful for biodiversity documentation and analysis. It is a powerful tool for the preparation of species database, atlases, derivative maps for identification of biological hotspots and preparation of habitat-wise conservation plans and for implementing national biodiversity strategies and action plans [1,2].

Conclusion: The information generated on Biodiversity sector could be used for a variety of purposes including to obtain an overview of forestry situation, to formulate policy decisions, to take strategic decisions in forestry and ecosystem management, to plan development programmes in forestry and ecosystem management.

Acknowledgements: The research has been supported Department of Biotechnology, New Delhi, India.

\section{References}

1. Jamison D.C. (2003) Bioinformatics. 12:679.

2. Soberón J., Peterson A.T. (2004) Bioinformatics. 29:1444. 